\title{
Estrutura trófica e ocupação de hábitat da avifauna de um parque urbano em Porto Alegre, Rio Grande do Sul, Brasil
}

\author{
Janete de Fátima Martins Scherer* \\ Angelo Luís Scherer \\ Maria Virginia Petry \\ Programa de Pós-Graduação em Diversidade e Manejo da Vida Silvestre \\ Laboratório de Ornitologia e Animais Marinhos \\ Universidade do Vale do Rio dos Sinos \\ Avenida Unisinos, 950, CEP 93022-000, São Leopoldo - RS, Brasil \\ *Autor para correspondência \\ netscherer@yahoo.com.br
}

Submetido em 02/06/2009

Aceito para publicação em 01/11/2009

\section{Resumo}

Durante o presente estudo foram registradas no PMMM 103 espécies, sendo 15,7\% residentes, 40,2\% prováveis residentes e 44,1\% ocasionais ou sobrevoantes. Onívoros e insetívoros somam 73,5\%. Área úmida e campo são os hábitats mais utilizados, seguido de mata ciliar. A riqueza difere significativamente entre hábitats e estações do ano, enquanto que na interação hábitats/estações do ano não diferiu. A abundância entre hábitats não diferiu significativamente, mas difere significativamente entre estações do ano e na interação hábitats/ estações do ano.

Unitermos: área úmida, área urbana, aves, conservação de aves, guilda alimentar

\section{Abstract}

Trophic structure and habitat occupation of avifauna in a Porto Alegre urban park. During the present study, 103 species were registered in the Parque Marechal Mascarenhas de Moraes (PMMM). Of these species, $15.7 \%$ were resident, $40.2 \%$ were probably resident, and $44.1 \%$ were occasional or overfliers. Omnivores and insectivores were the feeding guilds with the highest representation in the park, constituting $73.5 \%$ of the avifauna. The humid areas and fields were the habitats of the majority of the avifauna, followed by the ciliar forest. Comparing the richness of birds in the different habitats, significant differences were observed in the different seasons of the year during the study. However, regarding the interaction between habitats and seasons of the year, there were no significant differences. Comparing the abundances of birds in the different habitats, there were no significant differences, but they did differ significantly between seasons and also between the interactions of habitats and seasons.

Key words: birds, bird conservation, feeding guild, urban area, wetlands 


\section{Introdução}

Para o ano de 2025 se prevê que a população urbana dos países em desenvolvimento, incluindo os países do Neotrópico, chegará a quatro bilhões de habitantes (três vezes mais que a população urbana esperada para os países desenvolvidos), o que provocará um grande impacto sobre os ecossistemas naturais e seminaturais (Marsluff et al., 2001). Parques e reservas mantêm parcelas naturais da paisagem, de modo a preservar uma grande parte da vida natural das regiões onde são implantados (Wilson, 1997).

Os efeitos da urbanização sobre aves têm sido bastante pesquisados, sendo que diversos fatores complexamente interligados são considerados para explicar a redução verificada no estabelecimento de aves em áreas urbanas (Marzluff et al., 2001). Muitas aves buscam em parques urbanos não somente abrigo, mas também alimentação e locais para nidificação. Alguns autores consideram que parques e áreas verdes públicas, que compõem a vegetação urbana, são de grande importância para as aves, porém ressaltam que estes ambientes não são suficientes para propiciar refúgios (Sander e Voss, 1982a; Gilbert, 1989; Argel-de-Oliveira, 1996; Efe et al., 2001).

As aves merecem destaque por sua diversidade taxonômica, característica bioindicadora e grande capacidade de deslocar-se para escolha de seu hábitat, sendo que a avaliação de sua comunidade é uma importante ferramenta para verificar o grau de conservação e qualidade dos hábitats (Argel-de-Oliveira, 1995; Blair, 1999; Hermy e Cornelis, 2000). Estudos que buscam averiguar a diversidade deste grupo em áreas urbanas, principalmente em praças e parques, foram realizados por vários autores (Sander e Voss, 1982b; Ruszczyk et al., 1987; MatarazzoNeuberger, 1992; Argel-de-Oliveira, 1995; Mendonça-Lima e Fontana, 2000), tendo ainda trabalhos de cunho trófico (Matarazzo-Neuberger, 1995; Villanueva e Silva, 1996; Leveau e Leveau, 2004). No PMMM foram desenvolvidos estudos relacionados às guildas (Scherer et al., 2005), levantamentos de riqueza (Efe et al., 2001) e descrição de aspectos de nidificação (Scherer et al., 2006).

Segundo Root (1967), as guildas tróficas correspondem ao grupo de espécies que exploram a mesma classe de recursos de maneira semelhante. Neste sentido, a estrutura e composição das comunidades de aves sofrem mudanças quando ocorrem alterações na vegetação, sejam elas naturais ou provocadas por atividades humanas (Aleixo, 1999).

$\mathrm{O}$ conhecimento das exigências ecológicas de muitas famílias, gêneros e espécies de aves pode ser suficiente em diversas situações para indicar condições ambientais às quais são sensíveis. Portanto, alterações de vegetação implicam que o ambiente natural pode tornar-se impróprio para abrigar aves que exigem condições específicas para sobreviver. Desta forma, a paisagem torna-se uma das características ambientais mais importantes na composição da avifauna urbana (Argel-de-Oliveira, 1996). O objetivo do trabalho é contribuir para o inventário da avifauna de Porto Alegre, relacionando a avifauna do Parque Marechal Mascarenhas de Moraes e suas guildas tróficas aos hábitats existentes no local.

\section{Material e Métodos}

O Parque Marechal Mascarenhas de Moraes

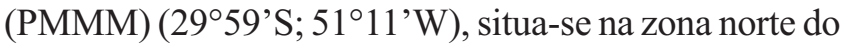
município de Porto Alegre - Rio Grande do Sul (Figura 1) e fica somente a $6,6 \mathrm{~km}$ do Aeroporto Internacional Salgado Filho. O local onde se encontra o PMMM era várzea do rio Gravataí, servindo de aterro sanitário até o ano de 1973. Após manejo e soterramento do lixo, criouse em 1982 um Parque Municipal. Atualmente o PMMM possui uma área total de 18,2ha dividida em: campos limpos (35\%), mata ciliar (21\%) e área úmida (44\%). Os campos limpos apresentam vegetação composta por gramíneas e árvores de grande porte, como Peltophorum dubium e Tipuana tipu (Caesalpiniaceae) e Enterolobium contortisiliquum (Mimosaceae). A área é utilizada pela população para lazer, pois nesta está localizado o playground e espaço para a prática de exercícios. A mata ciliar é composta principalmente por Erythrina cristagalli (Fabaceae), Inga sessilis (Mimosaceae), Tipuana tipu (Caesalpiniaceae) e vegetação sub-árborea, que embeleza parte das trilhas do local. Aárea úmida serve de local de reprodução para diversas espécies das famílias Ardeidae, Anatidae, Threskiornithidae, entre outras. Em 


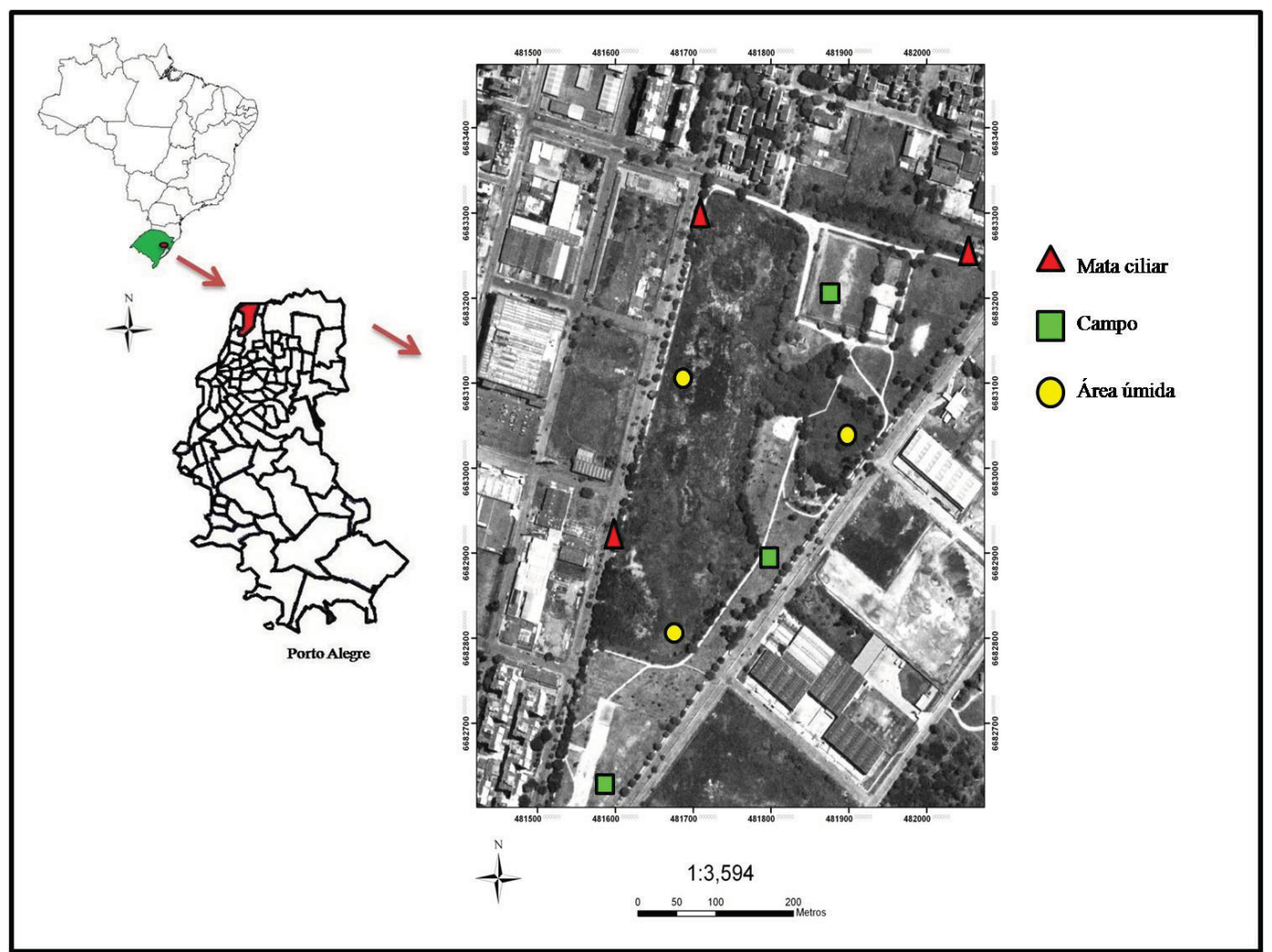

FIGURA 1: Pontos de observação na área de estudo do Parque Marechal Mascarenhas de Moraes, Porto Alegre, RS, Brasil.

períodos prolongados de estiagem, a área tem sua lâmina d'água baixa ou em grande parte inexistente, visto que seu tamanho é de aproximadamente 8 ha. A vegetação é composta predominantemente por Mimosa bimucronata (Mimosaceae), Juncus sp. (Juncaceae) e Eucalyptus sp. (Myrtaceae) secos, além de uma grande diversidade de macrófitas aquáticas.

Foram realizadas saídas a campo mensais no período entre janeiro de 2004 a outubro de 2006, totalizando $128 \mathrm{~h}$ de observações em 32 expedições. As observações foram realizadas em todos os pontos nas primeiras horas da manhã ( $75 \%$ delas) e no final da tarde, antes do pôr do sol (25\%), quando da maior atividade das aves. Na estação do ano, verão de 2005 , não foram realizadas observações. As aves observadas nos deslocamentos entre os pontos foram consideradas apenas para análise qualitativa.

Foram determinados três pontos de observação com distância mínima de 200m entre si em cada hábitat (área úmida, campo e mata ciliar), permitindo independência entre os mesmos. Em cada ponto dos três hábitats foram realizadas 32 contagens que tiveram duração de $15 \mathrm{~min}$ por ponto (Bibby et al., 2000). Utilizou-se um raio de $50 \mathrm{~m}$ no ponto a partir do observador para detectar as aves. As espécies foram identificadas através da visualização direta, com auxílio de binóculos ou através da vocalização, e os dados anotados em planilhas específicas. A nomenclatura e ordem taxonômica das espécies seguem CBRO (2008). As espécies foram classificadas nos hábitats área úmida, campo e mata ciliar, de acordo com a ocupação destes durante as observações no parque.

A frequência de ocorrência (FO) foi calculada a partir da relação do número de registros de cada espécie pelo número total de visitas (32) e classificada em categorias de ocupação, segundo Mendonça-Lima e Fontana (2000), adaptadas de Argel-de-Oliveira (1995): residente $(\mathrm{FO}>0,60)$, prováveis residentes $(0,60>\mathrm{FO}$ $\geq 0,15)$, ocasionais e/ou sobrevoantes $(\mathrm{FO}<0,15)$. O cálculo do índice de frequência de ocorrência seguiu Vielliard e Silva (1990), ou seja, somente foi considerado o número de visitas onde a espécie foi registrada, sendo este índice expresso em porcentagem. 
Os agrupamentos tróficos utilizados foram baseados em Willis (1979), Sick (1997), Belton (2000), e em observações pessoais, de acordo com as seguintes categorias: Insetívoros - alimentação baseada principalmente em insetos que podem ser capturados no chão, no ar entre a vegetação e na casca de árvores; Carnívoros - alimentação baseada em grandes invertebrados, pequenos e grandes vertebrados vivos; Frugívoros - alimentação baseada principalmente em frutos; Nectarívoros - alimentação baseada principalmente em néctar; Necrófagos - alimentação baseada em animais mortos; Onívoros - alimentação baseada em frutos, artrópodes e pequenos vertebrados; Granívoros - alimentação baseada na predação de sementes; Piscívoros - alimentação baseada em peixes.

Primeiramente foi realizado teste de normalidade dos dados de Kolmogorov Smirnov. Após foram testadas diferenças da riqueza e abundância de aves nos três hábitats e nas estações do ano através de ANOVA para medidas repetidas com o emprego do programa Systat 12 (Sokal e Rohlf, 1995).

O estado de conservação das espécies foi determinado com base em Birdlife Internacional (2008), Ministério do Meio Ambiente (2008) e Decreto ${ }^{\circ} 41.672$ de 10 de junho de 2002, disponível em Marques et al. (2002).

\section{Resultados}

Durante o presente estudo foram registradas no PMMM 103 espécies pertencentes a 33 famílias. Para as espécies é apresentado o hábitat, guilda alimentar, frequência de ocorrência e categoria de ocupação (Tabela 1). Destas espécies, $15,7 \%(\mathrm{n}=16)$ são residentes, $40,2 \%$ ( $\mathrm{n}=41)$ são prováveis residentes e 44,1\% $(\mathrm{n}=45)$ são ocasionais ou sobrevoantes. Das espécies ocasionais ou sobrevoantes, $80 \%$ possuem apenas um ou dois registros durante o período de estudo. Apenas nove espécies têm uma frequência de ocorrência maior que $75 \%$ (Tabela 1). A espécie Pardirallus maculatus foi registrada após o período de estudo, sendo considerada apenas na análise qualitativa.
Onívoros $(44,1 \%)$ e insetívoros $(29,4 \%)$ foram as guildas alimentares com maior representação no parque. As outras guildas representam apenas 26,5\% da avifauna, destacando-se granívoros $(10,8 \%)$, carnívoros $(6,9 \%)$ e frugívoros $(4,9 \%)$. Observa-se que campo e área úmida são os hábitats da maioria da avifauna, com 55 e 42 espécies respectivamente. Foram registradas 37 espécies exclusivas para o hábitat área úmida, sendo que 25 destas são onívoras. Para o hábitat campo foram 24 espécies exclusivas, onde oito são granívoras e seis onívoras. Foram observadas 12 espécies exclusivas para o habitat mata ciliar, que apresentou sete espécies insetívoras (Tabela 1).

Verificou-se que a riqueza entre os hábitats mata ciliar, campo e área úmida no PMMM, apresentou diferença significativa $(\mathrm{F}=23,68 ; \mathrm{gl}=2,6 ; \mathrm{p}=0,001)$, assim como nas diferentes estações do ano $(\mathrm{F}=8,55 ; \mathrm{gl}=9,54$; $\mathrm{p}<0,001$ ), sendo maior na primavera de 2005 (Figura 2). No entanto, não ocorreu diferença significativa da riqueza na interação hábitats e estações do ano $(\mathrm{F}=1,54$; $\mathrm{gl}=18,54 ; \mathrm{p}>0,05)$.

A abundância nos três hábitats não apresentou diferença significativa $(F=2,92 ; \mathrm{gl}=2,6 ; \mathrm{p}>0,05)$. No entanto, entre as estações do ano ocorreu diferença significativa da abundância $(\mathrm{F}=2,91 ; \mathrm{gl}=9,54 ; \mathrm{p}=0,007)$, assim como na interação hábitats e estações do ano $(\mathrm{F}=2,75 ; \mathrm{gl}=18,54 ; \mathrm{p}=0,001)$ (Figura 3). O inverno de 2004 apresentou a maior abundância com 865 indivíduos, sendo em sua maioria de Ardea alba e Dendrocygna viduata, enquanto outono de 2004 apresentou a menor abundância com 131 indivíduos. Observaram-se em média 243 indivíduos por visita, sendo que no mês de setembro de 2004 ocorreu a maior abundância com 734 indivíduos. As espécies com maior abundância em uma única visita foram Ardea alba $(\mathrm{n}=480)$ em setembro de 2004 e Egretta thula $(\mathrm{n}=183)$ em janeiro de 2004, em virtude destas espécies utilizarem a área úmida para nidificação. Neste contexto, verificou-se que $28,2 \%$ das espécies de aves do parque utilizam a área úmida para reprodução, destacando-se Phimosus infuscatus, Plegadis chihi, Egretta thula, Ardea alba, Nycticorax nycticorax, Bubulcus ibis, Chrysomus ruficapillus e Aramides saracura com grandes abundâncias. 
TABELA 1: Espécies de aves registradas no Parque Marechal Mascarenhas de Moraes em Porto Alegre, RS, Brasil, entre janeiro de 2004 e outubro de 2006.

\begin{tabular}{|c|c|c|c|c|}
\hline Família/ Espécie & Hábitat no PMMM & Guilda & FO & $\mathrm{CO}$ \\
\hline \multicolumn{5}{|l|}{ ANHIMIDAE } \\
\hline Chauna torquata & Área úmida & $\mathrm{ON}$ & 0,13 & $\mathrm{O}$ \\
\hline \multicolumn{5}{|l|}{ ANATIDAE } \\
\hline Dendrocygna viduata* & Área úmida & ON & 0,75 & $\mathrm{R}$ \\
\hline Amazonetta brasiliensis & Área úmida & $\mathrm{ON}$ & 0,09 & $\mathrm{O}$ \\
\hline \multicolumn{5}{|l|}{ PHALACROCORACIDAE } \\
\hline Phalacrocorax brasilianus & Área úmida & PI & 0,03 & $\mathrm{O}$ \\
\hline \multicolumn{5}{|l|}{ ARDEIDAE } \\
\hline Ixobrychus involucris & Área úmida & $\mathrm{ON}$ & 0,03 & $\mathrm{O}$ \\
\hline Nycticorax nycticorax* & Área úmida & $\mathrm{ON}$ & 0,41 & $\mathrm{P}$ \\
\hline Butorides striata* & Área úmida & ON & 0,16 & $\mathrm{P}$ \\
\hline Bubulcus ibis* & Área úmida & $\mathrm{ON}$ & 0,28 & $\mathrm{P}$ \\
\hline Ardea cocoi & Área úmida & $\mathrm{ON}$ & 0,03 & $\mathrm{O}$ \\
\hline Ardea alba* & Área úmida & $\mathrm{ON}$ & 0,75 & $\mathrm{R}$ \\
\hline Syrigma sibilatrix & Área úmida & $\mathrm{ON}$ & 0,06 & $\mathrm{O}$ \\
\hline Egretta thula* & Área úmida & $\mathrm{ON}$ & 0,28 & $\mathrm{P}$ \\
\hline \multicolumn{5}{|l|}{ THRESKIORNITHIDAE } \\
\hline Plegadis chihi* & Área úmida & $\mathrm{ON}$ & 0,34 & $\mathrm{P}$ \\
\hline Phimosus infuscatus* & Área úmida & $\mathrm{ON}$ & 0,59 & $\mathrm{P}$ \\
\hline \multicolumn{5}{|l|}{ CATHARTIDAE } \\
\hline Coragyps atratus & Campo & $\mathrm{NC}$ & 0,06 & $\mathrm{O}$ \\
\hline \multicolumn{5}{|l|}{ ACCIPITRIDAE } \\
\hline Rostrhamus sociabilis & Área úmida & $\mathrm{CA}$ & 0,06 & $\mathrm{O}$ \\
\hline Circus buffoni & Área úmida & $\mathrm{CA}$ & 0,06 & $\mathrm{O}$ \\
\hline Rupornis magnirostris & Mata ciliar & CA & 0,06 & $\mathrm{O}$ \\
\hline \multicolumn{5}{|l|}{ FALCONIDAE } \\
\hline Caracara plancus & Área úmida e campo & CA & 0,16 & $\mathrm{P}$ \\
\hline Milvago chimango & Campo & $\mathrm{ON}$ & 0,16 & $\mathrm{P}$ \\
\hline Micrastur semitorquatus & Área úmida & $\mathrm{CA}$ & 0,03 & $\mathrm{O}$ \\
\hline Falco sparverius & Área úmida e campo & $\mathrm{CA}$ & 0,03 & $\mathrm{O}$ \\
\hline \multicolumn{5}{|l|}{ ARAMIDAE } \\
\hline Aramus guarauna & Área úmida & $\mathrm{CA}$ & 0,06 & $\mathrm{O}$ \\
\hline \multicolumn{5}{|l|}{ RALLIDAE } \\
\hline Aramides cajanea & Área úmida & $\mathrm{ON}$ & 0,16 & $\mathrm{P}$ \\
\hline Aramides saracura* & Área úmida e mata ciliar & $\mathrm{ON}$ & 0,16 & $\mathrm{P}$ \\
\hline Laterallus melanophaius & Área úmida & $\mathrm{ON}$ & 0,09 & $\mathrm{O}$ \\
\hline Pardirallus maculatus & Área úmida & $\mathrm{ON}$ & - & - \\
\hline Pardirallus nigricans & Área úmida & $\mathrm{ON}$ & 0,16 & $\mathrm{P}$ \\
\hline Pardirallus sanguinolentus & Área úmida & $\mathrm{ON}$ & 0,09 & $\mathrm{O}$ \\
\hline Gallinula chloropus* & Área úmida & ON & 0,94 & $\mathrm{R}$ \\
\hline \multicolumn{5}{|l|}{ CHARADRIIDAE } \\
\hline Vanellus chilensis* & Área úmida e campo & ON & 1,00 & $\mathrm{R}$ \\
\hline \multicolumn{5}{|l|}{ JACANIDAE } \\
\hline Jacana jacana* & Área úmida & $\mathrm{ON}$ & 0,97 & $\mathrm{R}$ \\
\hline
\end{tabular}




\begin{tabular}{|c|c|c|c|c|}
\hline LARIDAE & & & & \\
\hline Chroicocephalus sp. & Área úmida & ON & 0,03 & $\mathrm{O}$ \\
\hline COLUMBIDAE & & & & \\
\hline Columbina talpacoti* & Campo & GR & 0,50 & $\mathrm{P}$ \\
\hline Columbina picui* & Campo & GR & 0,81 & $\mathrm{R}$ \\
\hline Columba livia* & Campo & ON & 0,47 & $\mathrm{P}$ \\
\hline Zenaida auriculata & Campo & GR & 0,41 & $\mathrm{P}$ \\
\hline Leptotila verreauxi & Mata ciliar & GR & 0,03 & $\mathrm{O}$ \\
\hline CUCULIDAE & & & & \\
\hline Crotophaga ani & Área úmida e mata ciliar & ON & 0,16 & $P$ \\
\hline Guira guira & Campo & ON & 0,66 & $\mathrm{R}$ \\
\hline TROCHILIDAE & & & & \\
\hline Chlorostilbon lucidus & Campo e mata ciliar & NE & 0,09 & $\mathrm{O}$ \\
\hline Hylocharis chrysura & Campo e mata ciliar & NE & 0,63 & $\mathrm{R}$ \\
\hline PICIDAE & & & & \\
\hline Colaptes melanochloros & Campo e área úmida & IN & 0,19 & $\mathrm{P}$ \\
\hline Colaptes campestris & Campo & IN & 0,38 & $P$ \\
\hline THAMNOPHILIDAE & & & & \\
\hline Mackenziaena leachii & Mata ciliar & IN & 0,03 & $\mathrm{O}$ \\
\hline FURNARIIDAE & & & & \\
\hline Furnarius rufus* & Área úmida e campo & ON & 0,94 & $\mathrm{R}$ \\
\hline Spartonoica maluroides & Área úmida & $\mathrm{IN}$ & 0,03 & $\mathrm{O}$ \\
\hline Schoeniophylax phryganophilus & Área úmida & IN & 0,13 & $\mathrm{O}$ \\
\hline Synallaxis spixi & Área úmida e mata ciliar & IN & 0,28 & $P$ \\
\hline Certhiaxis cinnamomeus $*$ & Área úmida & IN & 0,78 & $\mathrm{R}$ \\
\hline Anumbius annumbi & Campo & IN & 0,16 & $P$ \\
\hline Syndactyla rufosuperciliata & Mata ciliar & IN & 0,03 & $\mathrm{O}$ \\
\hline TYRANNIDAE & & & & \\
\hline Elaenia flavogaster & Mata ciliar & ON & 0,34 & $\mathrm{P}$ \\
\hline Elaenia sp. & Mata ciliar & ON & 0,03 & $\mathrm{O}$ \\
\hline Camptostoma obsoletum & Mata ciliar & IN & 0,03 & $\mathrm{O}$ \\
\hline Serpophaga nigricans & Área úmida & IN & 0,03 & $\mathrm{O}$ \\
\hline Serpophaga subscritata & Mata ciliar & IN & 0,34 & $\mathrm{P}$ \\
\hline Capsiempis flaveola & Mata ciliar & IN & 0,06 & $\mathrm{O}$ \\
\hline Phylloscartes ventralis & Mata ciliar & IN & 0,09 & $\mathrm{O}$ \\
\hline Platyrinchus mystaceus & Mata ciliar & IN & 0,03 & $\mathrm{O}$ \\
\hline Pyrocephalus rubinus & Área úmida & IN & 0,19 & $P$ \\
\hline Satrapa icterophrys & Área úmida & IN & 0,25 & $\mathrm{P}$ \\
\hline Xolmis irupero & Campo & IN & 0,34 & $P$ \\
\hline Machetornis rixosa & Campo & $\mathrm{IN}$ & 0,44 & $P$ \\
\hline Pitangus sulphuratus* & Campo, mata ciliar e área úmida & ON & 0,97 & $\mathrm{R}$ \\
\hline Empidonomus varius & Campo e mata ciliar & $\mathrm{IN}$ & 0,03 & $\mathrm{O}$ \\
\hline Tyrannus melancholicus & Campo e mata ciliar & IN & 0,38 & $\mathrm{P}$ \\
\hline Tyrannus savana & Campo e mata ciliar & IN & 0,22 & $\mathrm{P}$ \\
\hline HIRUNDINIDAE & & & & \\
\hline Tachycineta leucorrhoa & Campo e área úmida & IN & 0,28 & $\mathrm{P}$ \\
\hline Tachycineta meyeni & Campo e área úmida & IN & 0,34 & $\mathrm{P}$ \\
\hline
\end{tabular}




\begin{tabular}{|c|c|c|c|c|}
\hline Progne tapera* & Campo & IN & 0,53 & $\mathrm{P}$ \\
\hline Pygochelidon cyanoleuca* & Campo e área úmida & IN & 0,84 & $\mathrm{R}$ \\
\hline \multicolumn{5}{|l|}{ TROGLODYTIDE } \\
\hline Troglodytes musculus* & Campo e mata ciliar & ON & 0,94 & $\mathrm{R}$ \\
\hline \multicolumn{5}{|l|}{ POLIOPTILIDAE } \\
\hline Polioptila dumicola & Área úmida e mata ciliar & IN & 0,03 & $\mathrm{O}$ \\
\hline \multicolumn{5}{|l|}{ TURDIDAE } \\
\hline Turdus rufiventris* & Campo, mata ciliar e área úmida & ON & 0,72 & $\mathrm{R}$ \\
\hline Turdus amaurochalinus* & Campo, mata ciliar e área úmida & $\mathrm{ON}$ & 0,25 & $\mathrm{P}$ \\
\hline Turdus albicollis & Área úmida e mata ciliar & $\mathrm{ON}$ & 0,06 & $\mathrm{O}$ \\
\hline \multicolumn{5}{|l|}{ MIMIDAE } \\
\hline Mimus saturninus* & Campo & $\mathrm{ON}$ & 0,47 & $\mathrm{P}$ \\
\hline Mimus triurus & Campo & ON & 0,06 & $\mathrm{O}$ \\
\hline \multicolumn{5}{|l|}{ COEREBIDAE } \\
\hline Coereba flaveola* & Campo e mata ciliar & IN & 0,44 & $\mathrm{P}$ \\
\hline \multicolumn{5}{|l|}{ THRAUPIDAE } \\
\hline Thraupis sayaca & Campo & FR & 0,44 & $\mathrm{P}$ \\
\hline Thraupis palmarum & Campo & FR & 0,06 & $\mathrm{O}$ \\
\hline Thraupis bonariensis & Campo & FR & 0,06 & $\mathrm{O}$ \\
\hline \multicolumn{5}{|l|}{ EMBERIZIDAE } \\
\hline Zonotrichia capensis & Campo & GR & 0,06 & $\mathrm{O}$ \\
\hline Poospiza nigrorufa & Área úmida & $\mathrm{ON}$ & 0,03 & $\mathrm{O}$ \\
\hline Poospiza lateralis & Mata ciliar & ON & 0,03 & $\mathrm{O}$ \\
\hline Sicalis flaveola* & Campo & GR & 0,38 & $\mathrm{P}$ \\
\hline Sicalis luteola & Campo & GR & 0,06 & $\mathrm{O}$ \\
\hline Coryphospingus cucullatus & Mata ciliar & GR & 0,03 & $\mathrm{O}$ \\
\hline Gubernatrix cristata & Campo & GR & 0,06 & $\mathrm{O}$ \\
\hline Paroaria coronata & Área úmida & GR & 0,03 & $\mathrm{O}$ \\
\hline \multicolumn{5}{|l|}{ PARULIDAE } \\
\hline Parula pitiayumi & Campo e mata ciliar & IN & 0,06 & $\mathrm{O}$ \\
\hline Geothlypis aequinoctialis & Área úmida e mata ciliar & IN & 0,44 & $\mathrm{P}$ \\
\hline \multicolumn{5}{|l|}{ ICTERIDAE } \\
\hline Icterus cayanensis* & Mata ciliar & $\mathrm{ON}$ & 0,03 & $\mathrm{O}$ \\
\hline Amblyramphus holosericeus & Área úmida & $\mathrm{ON}$ & 0,16 & $\mathrm{P}$ \\
\hline Chrysomus ruficapillus & Área úmida & ON & 0,56 & $\mathrm{P}$ \\
\hline Agelaioides badius & Área úmida e campo & $\mathrm{ON}$ & 0,22 & $\mathrm{P}$ \\
\hline Molothrus rufoaxillaris & Área úmida & $\mathrm{ON}$ & 0,03 & $\mathrm{O}$ \\
\hline Molothrus bonariensis & Área úmida e campo & $\mathrm{ON}$ & 0,63 & $\mathrm{R}$ \\
\hline \multicolumn{5}{|l|}{ FRINGILLIDAE } \\
\hline Euphonia chlorotica & Campo & FR & 0,22 & $\mathrm{P}$ \\
\hline Euphonia cyanocephala & Campo & FR & 0,03 & $\mathrm{O}$ \\
\hline \multicolumn{5}{|l|}{ ESTRILDIDAE } \\
\hline Estrilda astrild* & Campo & GR & 0,25 & $\mathrm{P}$ \\
\hline \multicolumn{5}{|l|}{ PASSERIDAE } \\
\hline Passer domesticus* & Campo & $\mathrm{ON}$ & 0,84 & $\mathrm{R}$ \\
\hline
\end{tabular}

Guilda alimentar: Insetívoro (IN), Onívoro (ON), Carnívoro (CA), Frugívoro (FR), Nectarívoro (NE), Necrófago (NC), Granívoro (GR), Piscívoro (PI). Frequência de ocorrência (FO). Categoria de Ocupação (CO): Residente (R), Provável residente (P), Ocasional e/ou sobrevoante $(\mathrm{O})$. Evidência de nidificação $(*)$. 


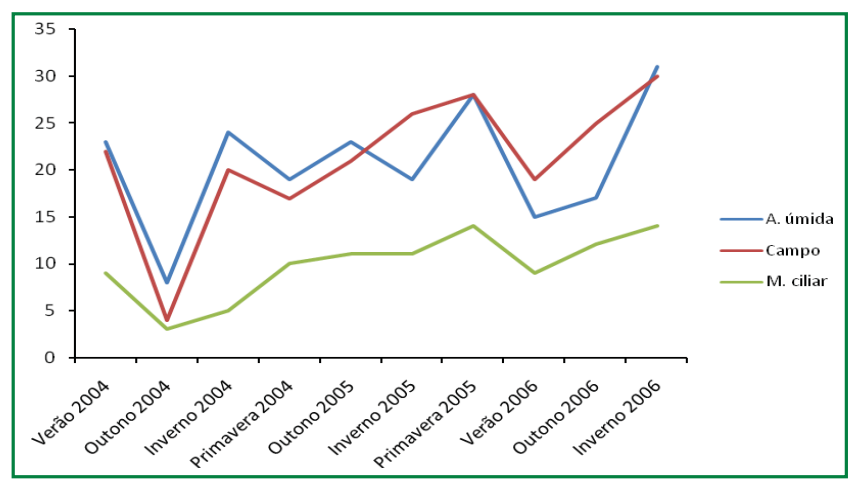

FIGURA 2: Riqueza máxima de aves registrada em cada hábitat por estação do ano no Parque Marechal Mascarenhas de Moraes, Porto Alegre, RS.

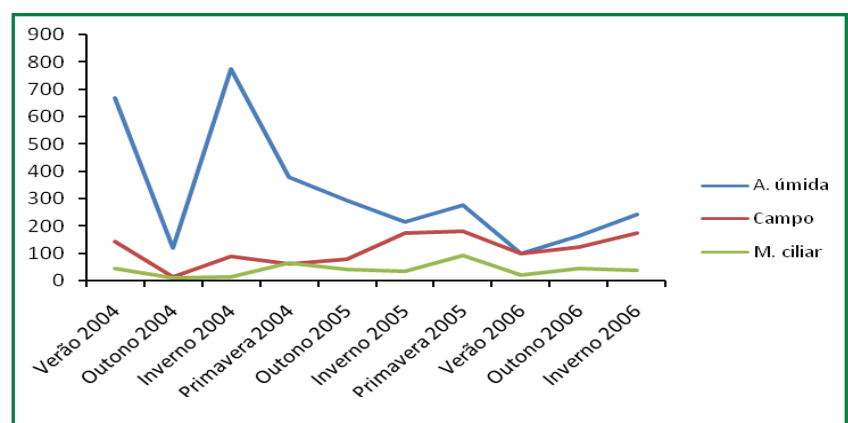

FIGURA 3: Abundância de aves registrada em cada hábitat por estação do ano no Parque Marechal Mascarenhas de Moraes, Porto Alegre, RS.

Como espécies de interesse conservacionista foram registradas Spartonoica maluroides, que é considerada "Quase ameaçada" em nível Mundial e "Vulnerável" no Rio Grande do Sul, Capsiempis flaveola, "Vulnerável" no Rio Grande do Sul, além de Gubernatrix cristata que está "Em perigo" em nível Mundial, Brasil e Rio Grande do Sul.

A curva de acumulação de espécies indica tendência a estabilizar a partir do $22^{\circ}$ mês, ocorrendo novos aumentos a partir do $29^{\circ}$ mês até o final do estudo, indicando que a assítota não foi atingida (Figura 4).

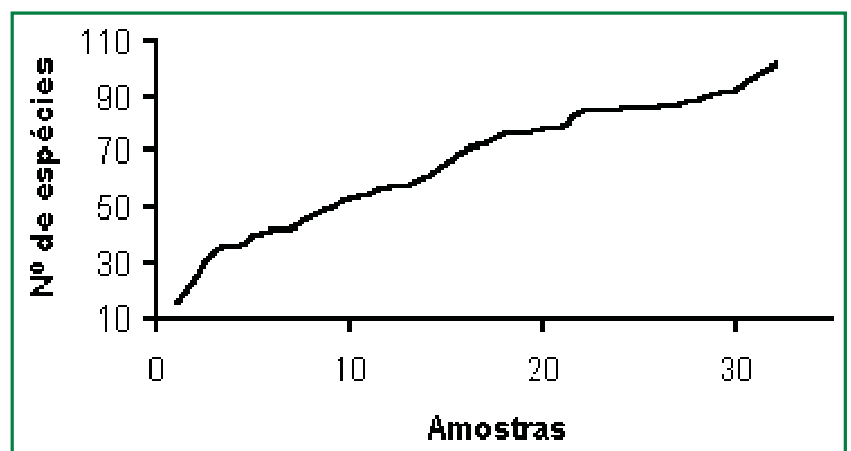

FIGURA 4: Curva de acumulação de espécies de aves do Parque Marechal Mascarenhas de Moraes, Porto Alegre, RS.

\section{Discussão}

A maior porcentagem de aves ocasionais ou sobrevoantes $(44,1 \%)$ está relacionada ao tamanho do parque, seu estado de conservação e também à proximidade do parque com o Lago Guaíba e com o Delta do Jacuí, que apresentam remanescentes de vegetação nativa, ajudando a manter a constituição da avifauna num estado mais conservado e permite influxo de espécies destes locais. Para Lancaster e Rees (1979), os migrantes parecem responder a características ambientais similares a seus ambientes de vida quando passam por uma região urbanizada. Desta forma, torna-se também mais lento atingir a assíntota da riqueza de aves na área de estudo. No PMMM, foram registradas 37 espécies exclusivas de área úmida, o que representa $35 \%$ do total e está situado no meio dessas áreas, principalmente próximo ao rio Gravataí. Accordi et al. (2001) registraram quase um terço das espécies de aves do delta nas áreas úmidas, enquanto Accordi e Barcellos (2006) registraram 283 espécies nas áreas úmidas da bacia hidrográfica do Lago Guaíba. Isto reforça que o parque pode servir de corredor entre as áreas úmidas do delta e região do Guaíba, pois também oferece ambiente propicio para a instalação de uma avifauna com hábitos aquáticos.

As espécies onívoras que podem utilizar o alimento de acordo com as condições oferecidas e as insetívoras que possuem disponibilidade de alimento $\mathrm{o}$ ano inteiro tendem a predominar no parque $(44 \% \mathrm{e}$ $29 \%$, respectivamente). A maior presença de onívoros e insetívoros também foi verificada por Scherer et al. (2005) nos oito parques de Porto Alegre, e em outros estudos realizados no Brasil (Argel-de-Oliveira, 1995; Villanueva e Silva, 1996; Valadão et al., 2006). É esperada a alta frequência de ocorrência de onívoros em fragmentos pequenos, pois a onivoria tem efeito tampão contra flutuações na disponibilidade de alimento nestes ambientes (Willis, 1979), enquanto que a alta porcentagem de espécies de aves insetívoras é padrão para a região tropical (Sick, 1997).

A baixa frequência de ocorrência de frugívoros no parque $(4,9 \%)$ e a sua limitada abundância podem estar relacionadas à indisponibilidade de alimento em diferentes épocas do ano em virtude da pouca arborização 
do parque e pequena mata ciliar. As alterações ambientais podem levar a uma tendência ao aumento de aves onívoras e possivelmente de insetívoros menos especializados e decréscimo de frugívoras e insetívoros mais especializados (Willis, 1979; Motta-Júnior, 1990; Vilanueva e Silva, 1996; Santos, 2004).

As espécies registradas no Parque Marechal Mascarenhas de Moraes representam 16,3\% da avifauna registrada para o Rio Grande do Sul (Bencke, 2001). Scherer et al. (2005) registraram 170 espécies para os oito parques de Porto Alegre e 83 espécies de aves para o mesmo parque. Entretanto, os autores não registraram em seu trabalho as espécies Chauna torquata, Circus buffoni, Pardirallus maculatus, Laterallus melanophaius, Aramides cajanea, Icterus cayanensis, Amblyramphus holosericeus, Euphonia cyanocephala, Molothrus rufoaxillaris para nenhum dos oito parques de Porto Alegre. Segundo lista de Fontana (2005), essas espécies são registradas em outros locais da cidade, mas também não são listadas para estes parques. Podem ser considerados novos registros para Porto Alegre as espécies Gubernatrix cristata, Tachycineta meyeni, Micrastur semitorquatus, Spartonoica maluroides e Capsiempis flaveola, visto que não constam em nenhuma lista.

Entre as espécies de interesse conservacionista ou com dados insuficientes de sua distribuição e ocorrência no Estado, destacam-se:

- Gubernatrix cristata registrado nos meses de outubro e novembro de 2005 , se alimentando na área de campo e provavelmente escapou de um cativeiro, pois era manso, permitindo aproximação. Segundo a Birdlife International (2008), esta espécie, endêmica dos Campos Sulinos, é classificada "em perigo" na lista da fauna ameaçada mundialmente, devido principalmente ao tráfico, perda de hábitat e plantações de Eucalyptus sp.

- Spartonoica maluroides ocorre sob status ocasional ou sobrevoante no PMMM e segundo a Birdlife International (2008), está "quase ameaçada" devido ao desaparecimento do seu hábitat de áreas úmidas com juncos, os quais são drenados e cultivados com plantações de Eucalyptus sp. e Pinus sp. Existe uma grande área de juncos no parque, o que proporciona um habitat favorável, pois devido à altura des- ta vegetação, a ave fica protegida contra possíveis predadores, dificultando a visualização. A espécie é residente e nidificante no Uruguai, sendo sua visualização pouco comum (Aspiroz, 2006) e no Brasil ocorre apenas no Rio Grande do Sul (Birdlife International, 2009).

- Capsiempis flaveola está na categoria "Least Concern" devido à sua população não estar globalmente quantificada. No Rio Grande do Sul, a espécie está "vulnerável", devido à perda de hábitat nas áreas úmidas, sendo sua distribuição entre as reservas florestais do Turvo e Nonoai (Belton, 2000). Na área de estudo aparece como status ocasional e/ou sobrevoante e foi observada na mata ciliar, indicando que este hábitat também pode ser utilizado pela espécie. É considerada uma espécie residente de primavera e nidifica no Rio Grande do Sul (Bencke, 2001).

- Micrastur semitorquatus não tem sua distribuição muito bem conhecida no Rio Grande do Sul e seu status é desconhecido (Bencke, 2001). Belton (2000) apresenta uma tímida distribuição da espécie na região do município de Taquara. Esta espécie costuma fazer seus ninhos em ocos de árvores (Thorstrom et al., 2001), como também ocupa ninhos de arara azul, o que foi verificado no pantanal brasileiro (Guedes, 1993; 2004). No PMMM, a espécie foi vista sobre os troncos de eucaliptos secos existentes na área úmida, que podem servir de local para nidificação, no entanto, não ocorreram registros da construção de ninhos.

- Tachycineta meyeni está na categoria "Least Concern" segundo a Birdlife International (2008). No Uruguai, a espécie é visitante de inverno e comum em pastagens e pradarias, porém com pouca abundância (Aspiroz, 2006). No Rio Grande do Sul, a espécie é visitante migratória vinda do cone sul do continente (Bencke, 2001). Porém, devido à escassez de estudos sobre a espécie, sua distribuição não é suficientemente conhecida.

- Pardiralus maculatus é considerado residente anual no Rio Grande do Sul, mas seu status não é confirmado (Bencke, 2001). São poucas as informações sobre esta espécie no Estado, sendo sua distribuição aleatória, com registros de indivíduos na depressão central e no litoral (Belton, 2000). No PMMM, a espécie provavelmente seja residente, mas devido à pouca vocalização , à dificuldade de visualização e à presença de muitos juncos, foi pouco registrada durante o estudo. 
Foram registradas espécies exóticas que estão estabelecidas no parque, de forma a usá-lo como local de reprodução e alimentação, sendo:

- Passer domesticus e Columba livia, capazes de aproveitar os recursos oferecidos pelos humanos de forma direta, permitindo a aproximação, sendo comuns em outros centros urbanos do mundo (Argelde-Oliveira, 1996; Clergeau et al., 1998; Blair, 1999; Leveau e Leveau, 2004; Lim e Sodhi, 2004).

- Estrilda astrild, que é provável residente no PMMM, pode ser visualizada em bandos de até 20 indivíduos se alimentando na vegetação baixa da área úmida e, segundo Belton (2000), a espécie é registrada em Porto Alegre desde antes de 1966.

A maior abundância de aves no inverno/2004 está relacionada ao início da atividade reprodutiva da maioria das aves no hemisfério sul no final do inverno e início da primavera. Destacam-se as famílias Ardeidae e Anatidae, que chegam ao local durante o mês de setembro utilizando a área úmida para nidificação. A queda da abundância a partir de abril, quando do início do outono, se deve ao fim do período reprodutivo das aves, diminuição das atividades de vocalização e diminuição no deslocamento das mesmas (Donatelli et al., 2004).

Considera-se muito importante a área úmida do parque, pois um terço das espécies depende deste local para alimentação e reprodução, ou vivem associadas a este ambiente. Segundo Maltchik et al. (2003), áreas úmidas abrigam uma diversidade ímpar de espécies da fauna e flora e um alto número de processos ecológicos, sendo importante sua conservação também em ambientes urbanos. Estas servem de refúgio e segurança, impedindo que pessoas cheguem próximas às aves, de modo que elas selecionam este hábitat (Campbell, 2006). Espécies como Dendrocygna viduata, Bubulcus ibis, Ardea alba, Egretta thula, Phimosus infuscatus e Laterallus melanophaius podem ser visualizadas no PMMM somente na área úmida, pois a aproximação humana não é possível ou está dificultada.

As espécies de aves maiores são mais sensíveis à presença de humanos e animais, possuindo uma maior distância para alerta e vôo com a presença humana, ao contrário das aves menores, que são mais tolerantes permitindo uma maior aproximação (Holmes et al.,
1993; Gutzwiller et al., 1998; Fernández-Juricic et al., 2001; Campbell, 2006). Neste sentido, a presença da área úmida é um fator crucial para a presença de aves de maior porte no parque, assim como a ocorrência de espaços verdes que promovem a diversidade da fauna, o que requer um planejamento adequado dos administradores do parque e órgãos competentes, no sentido de preservar as áreas existentes, além de criar ou ampliar outras áreas.

\section{Agradecimentos}

À Secretaria Municipal do Meio Ambiente de Porto Alegre (SMAM) pela oportunidade do estudo no Parque; à Sandra Lia Gravana Zeferino, administradora do Parque Marechal Mascarenhas de Moraes, pelo apoio; à Juli Massia, pela versão do abstract; a Rafael Gomes de Moura, pela imagem de satélite.

\section{Referências}

Accordi, I. A.; Barcellos, A. 2006. Composição da avifauna em oito áreas úmidas da Bacia Hidrográfica do Lago Guaíba, Rio Grande do Sul. Revista Brasileira de Ornitologia 14 (2): 101-115.

Accordi, I. A.; Vélez, E.; Albuquerque, E. P. 2001. Lista anotada das aves do Parque Estadual do Delta do Jacuí, RS. Acta Biologica Leopoldensia, 23 (1): 69-81.

Aleixo, A. 1999. Efects of selective logging on a bird community in the Brazilian Atlantic forest. The Condor, 101: 537-548.

Argel-de-Oliveira, M. M. 1995. Aves e vegetação em um bairro residencial da cidade de São Paulo (São Paulo, Brasil). Revista Brasileira de Zoologia, 12 (1): 81-92.

Argel-de-Oliveira, M. M. 1996. Aves urbanas. Anais do V Congresso Brasileiro de Ornitologia, Campinas, Brasil, p.151.

Azpiroz, A. B. 2006. Aves del Uruguay: Lista e introducción a su biologia y conservación. Aves Uruguay-Gupeca, Montevidéo, Uruguay, 105pp.

Belton, W. 2000. Aves do Rio Grande do Sul: Distribuição e biologia. Unisinos, São Leopoldo, Brasil, 584pp.

Bencke, G. A. 2001. Lista de referência das aves do Rio Grande do Sul. Fundação Zoobotânica do Rio Grande do Sul, Porto Alegre, Brasil, 104 pp.

Bibby, C. J.; Burgess, N. D.; Hill, D. A. 2000. Bird census techniques. $2^{\text {nd }}$ ed. Academic Press, London, UK, 256pp.

Birdlife International. 2008. Espécies da fauna mundial ameaçada de extinção. Disponível em <http:www.birdlife.org/datazone/ species/index.html >. Acesso em 14 de novembro de 2008.

Birdlife International. 2009. Species factsheet: Spartonoica maluroides. Disponível em <http://www.birdlife.org/datazone/ species/index.html $>$. Acesso em 28 de maio de 2009. 
Blair, R. B. 1999. Birds and butterflies along an urban gradients Surrogate taxa for assessing biodiversity? Ecological Applications, 9 (1): 164-170.

Campbell, M. O. 2006. Urban parks as shared spaces? The utily of alert distances indicators of avian tolerance of humans in Stirling, Scotland. Area, 38 (3): 301-311.

CBRO - Comitê Brasileiro de Registros Ornitológicos. 2008. Lista das Aves do Brasil. Disponível em $<\mathrm{http}$ :www.cbro.orb.br $>$. Acesso em 11 de dezembro de 2008.

Clergeau, P.; Savard, J. P. L.; Mennechez, G.; Falardeau, G. 1998. Bird abundance and diversity along an urban-rural gradient: A comparative study betweem two citie on different continents. The Condor, 100: 413-425.

Donatelli, R. J.; Costa, T. V. V.; Ferreira, C. D. 2004. Dinâmica da avifauna em fragmento de mata na fazenda Rio Claro, Lençóis Paulista, São Paulo, Brasil. Revista Brasileira de Zoologia, 21 (1): 97-114.

Efe, M. A.; Mohr, L. V.; Bugoni, L. 2001. Guia Ilustrado das Aves dos Parques de Porto Alegre. PROAVES, SMAM, COPESUL, CEMAVE, Porto Alegre, Brasil, 144pp.

Fernández-Juricic, E.; Dolores, M.; Lucas, E. 2001. Alert distance as an alterantive measure of bird tolerance to human disturbance implications for park design. Environmental Conservation, 28 (28): 263-269.

Fontana, C. S. 2005. A ornitofauna em Porto Alegre no século XX: Status de ocorrência e conservação. Comunicações do Museu de Ciências e Tecnologia da PUC, Série Zoológica, 18 (2): 161-206.

Gilbert, O. L. 1989. The ecology of urban hábitats. Chapman and Hall, London, UK, 369pp.

Guedes, N. M. R. 1993. Nidificação do gavião-relógio (Micrastur semitorquatus) no Pantanal. Anais do III Congresso Brasileiro de Ornitologia, Pelotas, Brasil, p.53-62.

Guedes, N. M. R. 2004. Araras azuis: 15 anos de estudos no Pantanal. Anais do Simpósio sobre Recursos Naturais e SócioEconômicos do Pantanal, Corumbá, Brasil, p.57.

Gutzwiller, K. L.; Marcum, H. A.; Harvey, H. B.; Roth, J.; Anderson, S. H. 1998. Bird tolerance to human intrusion in Wyoming montane forests. Condor, 100: 519-527.

Hermy, M.; Cornelis, J. 2000. Towards a monitoring method and a number of multifaceted and hierachical biodiversity incatiors for urban and suburban parks. Landscape and Urban Planning, 49: $149-162$.

Holmes, T. L.; Knight, R. L.; Stegall, L.; Craig, G. R. 1993. Responses of wintering grassland raptors to human disturbance. Wildlife Society Bulletin, 21: 461-468.

Lancaster, R. K.; Rees, W. E. 1979. Bid communities and the structure of urban hábitats. Canadian Journal of Zoology, 57: 2358-2368.

Leveau, L. M.; Leveau, C. M. 2004. Comunidades de aves en un gradiente urbano de la ciudad de Mar del Plata, Argentina. Hornero, 19 (1): 13-21

Lim, H.; Sodhi, N. S. 2004. Responses of avian guilds to urbanisation in a tropical city. Landscape and Urban Planning, 66: 199-215.

Maltchik, L.; Bertoluci, V. D. M.; Erba, D. A. 2003. Inventário das áreas úmidas do município de São Leopoldo, Rio Grande do Sul, Brasil. Pesquisas Botânicas, 53: 79-88.
Marques, A. A. B.; Fontana, C. S.; Veles, E.; Bencke, G. A.; Schneider, M.; Reis, R. E. 2002. Lista das espécies da fauna ameaçada de extinção no Rio Grande do Sul. Publicações Avulsas da Fundação Zoobotânica, 11: 52.

Marzluff, J. M.; Bowman, R.; Donnelly, R. 2001. A historical perpective on urban bird reserch: trends, terms, and approaches. In: Marzluff, J. M., Bowman, R. e Donnelly, R. (Eds). Avian ecology and conservation in an urbanizing world. Kluwer Academie, Boston, USA, p.1-17.

Matarazzo-Neuberber, W. M. 1992. Avifauna urbana de dois municípios da grande São Paulo, SP (Brasil). Acta Biologica Paranaense, 21: 89-106.

Matarazzo-Neuberber, W. M. 1995. Comunidades de aves de cinco parques e praças da Grande São Paulo, Estado de São Paulo. Ararajuba, 3: 13-19.

Mendonça-Lima, A.; Fontana, C. S. 2000. Composição, frequência e aspectos biológicos da avifauna no Porto Alegre country clube, Rio Grande do Sul. Ararajuba, 8 (1): 1-8.

Ministério do Meio Ambiente. 2008. Espécies da fauna brasileira ameaçada de extinção. Disponível em <http:www.mma.gob.br/ port/sbf/index.cfm>. Acesso em 15 de dezembro de 2008.

Motta-Júnior, J. C. 1990. Estrutura trófica e composição das avifaunas de três ambientes terrestres na região central do estado de São Paulo. Ararajuba, 1: 65-71.

Root, R. 1967. The niche exploration pattern on the Blue-grey Gnatcatcher. Ecoloy Monographs, 37: 317-350.

Ruszczyk, A.; Rodrigues, J. J. S.; Roberts, T. M. T.; Bendati, M. M. A.; Del Pino, R. S.; Marques, J. C. V.; Melo, M. T. Q. 1987. Distribution patterns of eight bird species in the urbanization gradiente of Porto Alegre, Brazil. Ciência e Cultura, 39 (1): 141987.

Sander, M.; Voss, W. A. 1982a. Quatro notas sobre aves no Rio Grande do Sul, Brasil. Pesquisas, 33: 28.

Sander, M.; Voss, W. A. 1982b. Aves livres observadas no Parque Farroupilha, Porto Alegre. Pesquisas, 33: 3-15.

Santos, A. M. R. 2004. Comunidade de aves em remanescentes florestais secundários de uma área rural no sudeste do Brasil. Ararajuba, 12 (1): 43-51.

Scherer, A.; Scherer, S. B.; Bugoni, L.; Mohr, L. V.; Efe, M. A.; Harts, S. M. 2005. Estrutura trófica da avifauna em oito parques da cidade de Porto Alegre, Rio Grande do Sul, Brasil. Ornithologia, 1 (1): 25-32.

Scherer, J. F. M.; Scherer, A. L.; Petry, M. V.; Teixeira, É. C. 2006. Estudo da avifauna associada à área úmida situada no Parque Mascarenhas de Moraes, zona urbana de Porto Alegre (RS). Biotemas, 19 (1): 107-110.

Sick, H. 1997. Ornitologia Brasileira. Nova Fronteira, Rio de Janeiro, Brasil, 912pp.

Sokal, R. R.; Rohlf, F. J. 1995. Biometry: The principles of statistics in biological research. Freeman, New York, USA, $887 \mathrm{pp}$.

Thorstrom, R.; Morales, C. M.; Ramos, J. D. 2001. Fidelity to territory, nest site and mate, survivorship, and reproduction of two sympatric Forest-Falcons. Journal of Raptor Research, 35 (2): 98-106. 
Valadão, R. M.; Franchin, A. G.; Marçal Júnior, O. 2006. A avifauna no Parque Municipal Victorio Siquierolli, zona urbana de Uberlândia (MG). Biotemas, 19 (1): 81-91.

Vielliard, J. M. E.; Silva, W. R. 1990. Nova metodologia de levantamento quantitativo e primeiros resultados no interior de São Paulo. Anais do Encontro Nacional dos Anilhadores de Aves, 4: 117-151.

Villanueva, R. E. V.; Silva, M. 1996. Organização Trófica da Avifauna do Campus da Universidade Federal de Santa Catarina (UFSC), Florianópolis, SC. Biotemas, 9 (2): 57- 69.

Willis, E. O. 1979. The composition of avian communities in remanescent woodlots in southern Brazil. Papéis Avulsos de Zoologia, 33 (1): 1-25.

Wilson, O. E. 1997. Biodiversidade. Nova Fronteira, Rio de Janeiro, Brasil, 660pp. 